2nd Galileo-XuGuangqi Meeting

International Journal of Modern Physics: Conference Series

Vol. 12 (2012) 136-145

(C) World Scientific Publishing Company

DOI: $10.1142 / \mathrm{S} 2010194512006344$

\title{
TIME RESOLVED SPECTRA OF GRBS SIMULTANEOUSLY DETECTED WITH BATSE and BeppoSAX WFCs
}

\author{
FILIPPO FRONTERA \\ Physics Department, University of Ferrara, Via Saragat, 1 \\ 44100 Ferrara, Italy \\ frontera@fe.infn.it \\ LORENZO AMATI \\ INAF, IASF Bologna, Via Gobetti 101 \\ 40129 Bologna, Italy \\ amati@iasfbo.inaf.it \\ RUBEN FARINELLI \\ INAF, IASF Palermo, Via U. La Malfa 153 \\ 90146 Palermo, Italy \\ farinelli@ifc.inaf.it \\ CRISTIANO GUIDORZI \\ Physics Department, University of Ferrara, Via Saragat, 1 \\ 44100 Ferrara, Italy \\ guidorzi@fe.infn.it \\ RAFFAELLA LANDI \\ INAF, IASF Bologna, Via Gobetti 101 \\ 40129 Bologna, Italy \\ landi@iasfbo.inaf.it \\ LEV TITARCHUK \\ Physics Department, University of Ferrara, Via Saragat, 1 \\ 44100 Ferrara, Italy \\ titarchuk@fe.infn.it \\ JEAN J.M. IN 'T ZAND \\ Space Research Organization in the Netherlands, Sorbonnelaan 2, \\ 3584 CA Utrecht, The Netherlands \\ J.J.M.in.t.Zand@sron.nl
}

We will discuss preliminary results of a systematic investigation devoted to study the time resolved broad-band spectra of the prompt emission of a sample of GRBs. These events were simultaneously detected with the BeppoSAX WFCs and the BATSE instrument aboard CGRO. We will discuss the fit of these spectra with the Band function, with 
a blackbody plus a power-law model, and with a Comptonization model developed by Titarchuk \& Farinelli (2011, to be submitted).

Keywords: Gamma Ray Bursts; GRB prompt emission spectra; data analysis; interpretation.

\section{Introduction}

In spite of the huge advances in the knowledge of the GRB afterglow properties, the GRB phenomenon, in particular its central engine, is still poorly understood. Of crucial importance, it is recognized the study of the prompt emission, which is more directly connected with the original explosion.

Among the open issues concerning the prompt emission is the radiation emission mechanism(s) at work and its origin. The best fit of the prompt emission spectra (either time averaged or time resolved) is still an empirical function, the Band function ${ }^{1}$ :

$$
N(E)=A\left(\frac{E}{100 k e V}\right)^{\alpha} \exp \left(-E / E_{0}\right)
$$

if

$$
(\alpha-\beta) \cdot E_{0} \geq E
$$

and

$$
N(E)=A\left[\frac{(\alpha-\beta) E_{0}}{100 k e V}\right]^{\alpha-\beta} \exp (\beta-\text { alpha }) \cdot\left(\frac{E}{100 k e V}\right)^{\beta}
$$

if

$$
(\beta-\alpha) \cdot E_{0} \leq E
$$

where $\alpha$ and $\beta$ are the power law low-energy (below $\mathrm{E}_{0}$ ) and high-energy (above $\mathrm{E}_{0}$ ) photon indices, respectively, and $\mathrm{A}$ is the normalization parameter.

Physical spectral models, like an optically thin synchrotron shock model (e.g., Ref. 2), or synchrotron emission as a result of an electromagnetic outflow ${ }^{3}$, are still under discussion. For example, several time-resolved spectra, below the photon peak energy $E_{p}$ of the $E F(E)$ spectrum $\left(=E^{2} N(E)\right.$ ), are inconsistent with an optically thin synchrotron model. Indeed it is found that the slope below $E_{p}$ is higher than maximum expected value: $4 / 3$ for an instantaneous spectrum; $1 / 2$ when the particle cooling is taken into account ${ }^{4}$. A different process, at least at early times, should be at work.

A partially physical model recently tested to interpret the time resolved spectra measured with BATSE is that proposed by Ref. 5: the sum of a blackbody (BB) plus a power-law (PL). The results found are very satisfactory, apart from 
a PL photon index $\Gamma$ in several cases $<2$, that would imply a diverging energy flux at high energies. However the BATSE experiment covers only the hard X-/soft gamma-ray energy band (20-2000 keV). How does the model fit change if the energy band of analysis is extended downward to $2 \mathrm{keV}$ ? This test can be performed by merging together BATSE and BeppoSAX/WFC data for the few GRBs contemporarily observed with both instruments. Goal of our investigation is a systematic time resolved spectral analysis of the GRBs detected with the BeppoSAX WFCs plus BATSE experiment. In this way we can derive spectra in the energy band from 2 to $2000 \mathrm{keV}$, a band very scarcely explored. In addition to test the model by Ref. 5, we test a new GRB prompt emission model (GRBCOMP) developed by Titarchuk \& Farinelli (2011, to be submitted).

\section{The GRB sample}

The GRB sample, reported in Table 1, consists of GRBs simultaneously observed with the Wide Field Cameras (WFCs) aboard the BeppoSAX satellite and with the BATSE instrument aboard the Compton Gamma Ray Observatory. The instrumentation that detected these bursts is widely described in the literaure. For the BATSE experiment see, e.g., Ref. 6, for WFC, see Ref. 7. The BATSE spectra were taken from the Large Area Detectors (LADs), whose typical passband is 25-2000 $\mathrm{keV}$, while the WFCs operated in normal mode with 31 energy channels in 2-28 keV and $0.5 \mathrm{~ms}$ time resolution.

As can be seen from Table 1, for only the brightest GRBs (970111, 980329, 990123, 990510) it was possible to subdivide their light curves in a significant number of intervals and to derive, in each of these intervals, statistically significant spectra.

\section{Spectral models for spectral deconvolution}

In Figs. 1 and 2 we show the light curves of the 4 brightest GRBs and the intervals in which we performed the spectral analysis.

For the deconvolution of the time resolved count rate spectra we assumed three different input models. In addition to the Band function, we used the model adopted

Table 1. GRBs simulaneously detected with BATSE and WFCs

\begin{tabular}{ccc}
\hline GRB name & $\begin{array}{c}2-700 \mathrm{keV} \text { Fluence } \\
\left(\times 10^{-6} \mathrm{cgs}\right)\end{array}$ & Number of time resolved spectra \\
\hline 970111 & 36.2 & 10 \\
971214 & 6.87 & 3 \\
980329 & 37.53 & 5 \\
980519 & 10.16 & 2 \\
990123 & 205.12 & 25 \\
990510 & 15.8 & 11 \\
990907 & 8.91 & 3 \\
991030 & 3.27 & 2 \\
\hline
\end{tabular}


by Ref. 5 (BB plus PL), and the GRBCOMP model under development by Titarchuk \& Farinelli (2011). The main ingredients of the latter model are:

- bulk Comptonization of a soft $\mathrm{X}$-ray blackbody spectrum in a hightemperature and optical depth sub-relativistically expanding outflow, consequence of the star explosion

- later convolution of the bulk Comptonization spectrum with a brokenpowerlaw Green function, aimed to reproduce the apparently nonthermal high-energy emission observed in the GRB prompt phase

While the first step of the model, related to the sub-relativistic expansion of the hot shell, has a well specified underlying physical scenario, confirmed with aim of hydrodynamical simulations by ${ }^{11}$, the second part is left to be semi-phenomenological, because it is not straightforward to estimate which is are the plasma dynamical and physical properties once it leaves the star photosphere (e.g., terminal bulk Lorenzt facot $\Gamma$, possible non-thermal electron distribution because of shock particle acceleration) which may give rise to a Green function like that described in grbcomp. A Montecarlo code to investigate the emerging spectra in
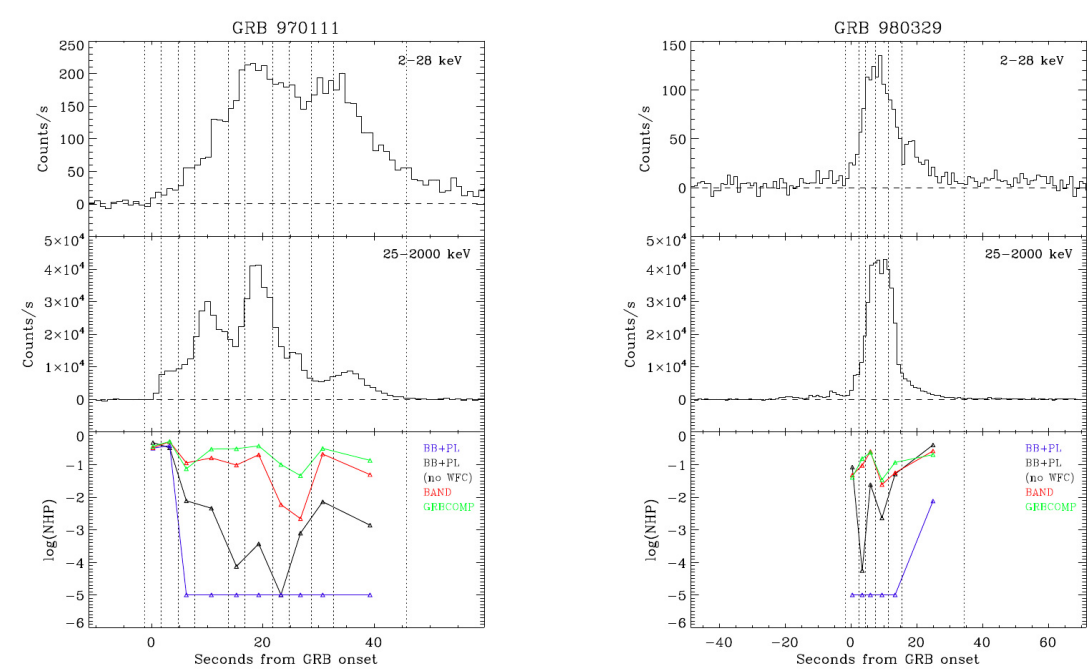

Fig. 1. Light curves of GRB 970111 and GRB 980329. The vertical dashed lines limit the regions in which the time resoved spectra have been derived. In the bottom panels, the null hypothesis probability of the best fits with diffrent models is shown. 
this phase is under developement. We note that a model consisting of a sub-relativistic and subsequent relativistic phase has also been proposed by $^{3}$, the electromagnetic fireball model, albeit in that case the the energy released comes from the Poynting vector. The current scenario of the GRBCOMP model is the explosion of a very massive star ( $\geq 130$ solar masses) by pair instability. With this assumption the energy budget is due to nuclear fusion. The explosion of such massive stars is expected in the early Universe, but recently it was found also in our Universe (e.g., Ref. 8).

In this scenario, the formation mechanism of the prompt emission spectrum is the following. First (up to the photospheric radius), a high-temperature plasma $\left(T \sim 10^{9} \mathbf{K}\right)$, consisting of both electrons and baryons, with very high optical depth, develops and moves outward with sub-relativistic bulk velocity $\left(V_{b}<0.5 \mathrm{c}\right)$, as a consequence of the star core explosion. Comptonization of soft seed photons presumably coming from the star photosphere and from the base of the hot shell $\left(k T_{\mathrm{bb}}<1-2\right.$ $\mathrm{keV}$ ) gives rise to a typical saturated Comptonization spectrum, which however deviates from a Wien-like shape because of the presence of the bulk outflow motion. As this hot outflow leaves the star photosphere, it
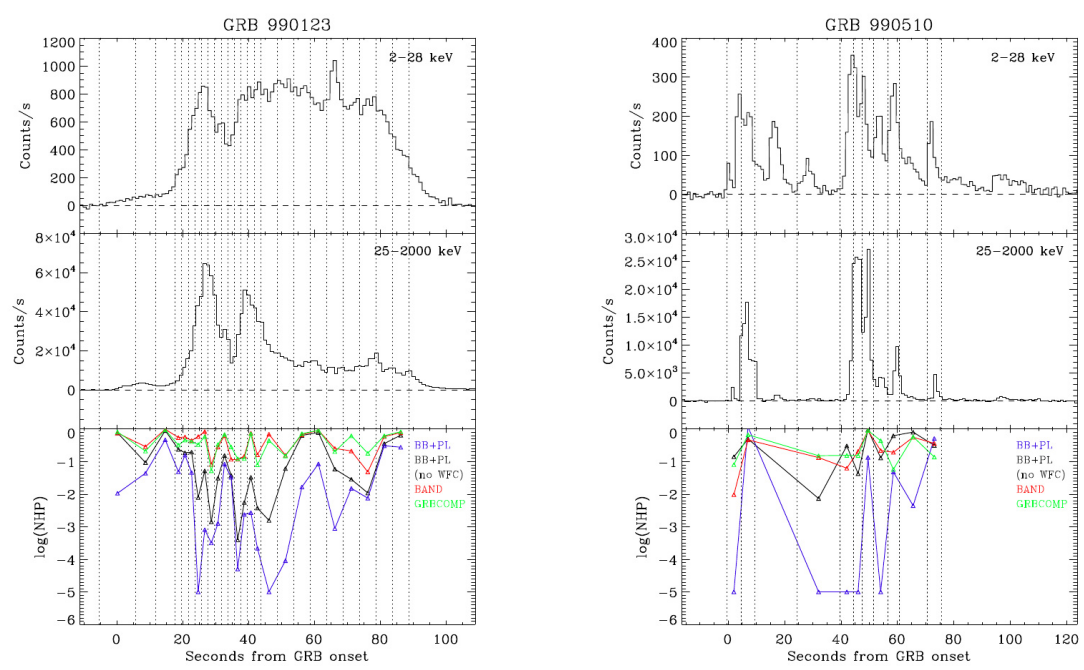

Fig. 2. Light curves of GRB 990123 and GRB 990510. The vertical dashed lines limit the regions in which the time resoved spectra have been derived. In the bottom panels, the null hypothesis probability of the best fits with diffrent models is shown. 
is subjected to a huge radiation pressure (more than $10^{10} L_{\mathrm{Edd}}$ ) coming from the underlyng star which may accelerate the matter up to relativistic velocities. Interaction with the interstellar medium and/or the star pre-ejecta may produce shock waves, with consequent particle acceleration and eventual non-thermal distributions. Albeit a quantitative treatment of this process is required, it is claimed that the interaction (inverse Compton) of the bulk Comptonized photons with non-thermal electrons moving at relativistic velocity produce the high-energy powerlaw observed in the prompt emission up to GeV. Mathematically, the spectral formation in this relativistic phase is decribed by mean of a convolution with a broken-powerlaw Green function.

The free parameters of the GRBCOMP model are the following:

(1) Seed photon temperature $k T_{s}$ related to the luminosity of the star;

(2) Electron temperature $k T_{e}$ of the plasma outflow;

(3) Bulk parameter

$$
\delta=\frac{2 \beta_{0}}{3 \tau_{e f f} \Theta}
$$

where $\beta_{0}=v / c$ is the bulk outflow velocity (assumed to be constant), $\tau_{\text {eff }}$ is the effective optical depth of the outflow bubble (optical depth averaged over the entire electron path) and $\Theta \equiv k T_{e} / m_{e} c^{2}$, with $m_{e}$ and $c$ being the electron mass and the light speed, respectively.

(4) Inverse of the Comptonization parameter $\gamma \propto 1 / Y$, with $\gamma \sim 0$ for saturated Comptonization;

(5) High-energy index $\alpha_{b}$ of the up-scattering Green function, related to the photon index $\beta$ of the Band low by $\alpha=-\beta+1$;

(6) Spectrum normalization $N$ given by

$$
N=R_{9}^{2} / D_{\mathrm{Mpc}}^{2}
$$

where $R_{9}$ is the apparent photospheric radius in units of $10^{9} \mathrm{~cm}$ and $D_{\mathrm{Mpc}}$ is the source distance in Mpc.

\section{Results}

In Figs. 1 and 2 we show the best fit acceptance level of the tested models, in terms of null hypothesis probability. As can be seen, the Band fucntion gives a good description of all time resolved spectra. Instead, apart from some time interval (specially at very early time and during the tail of the event), the PL + BB model does not give an acceptable description of the WFC plus BATSE time resolved spectra. However the best fits become acceptable when the spectral analysis is limited to the BATSE data ( $>20 \mathrm{keV})$, consistently with the findings by Ref. ${ }^{5}$. This result shows the importance of the broad band spectroscopy down to $\sim 1 \mathrm{keV}$ to test models. But the most important finding of our analysis is that the GRBCOMP 
model gives the best description of all the time resolved spectra, even better than the Band function. Given this analysis result, we have investigated its consequences. In a paper in preparation (Frontera et al. 2011) we will discuss this topic with details. Here we limit our considerations to some relevant results.

We find that the electron temperature $k T_{e}$ of the GRBCOMP model is correlated with the peak energy $E_{p}$ of the $E F(E)$ spectrum, derived with the Band function. The correlation is generally positive but in the case of GRB 980329 we fing a negative correlation. As an example, we show in Fig. 3 the correlation between $k T_{e}$ and $E_{p}$ in the case of GRB 970111.

This result could be a hint of the fact that other parameters contribute to the determination of the peak energy of $E F(E)$ spectrum. According to the GRBCOMP model, this parameter is the bulk parameter $\delta$. Indeed we find that the $k T_{e} / E_{p}$ ratio is correlated with $\delta$ (see, e.g., Fig. 4).

We also find that $k T_{e}$ is positively correlated with flux (Frontera et al., in preparetion). Taking into account the $k T_{e}$ correlation with $E_{p}$, the consequence is that $E_{p}$ is positively correlated with flux. This result is indeed found. Given that for each GRB the redshift correction is not needed, it strenghtens the robustness of the $E_{p}-E_{i s o}$ correlation $^{12}$.

As expected by the model, we do not find any correlation between $k T_{e}$ and the thermal temperature $k T_{s}$ of the seed photons, that are Comptonized by the plasma outflow (see Fig. 5).

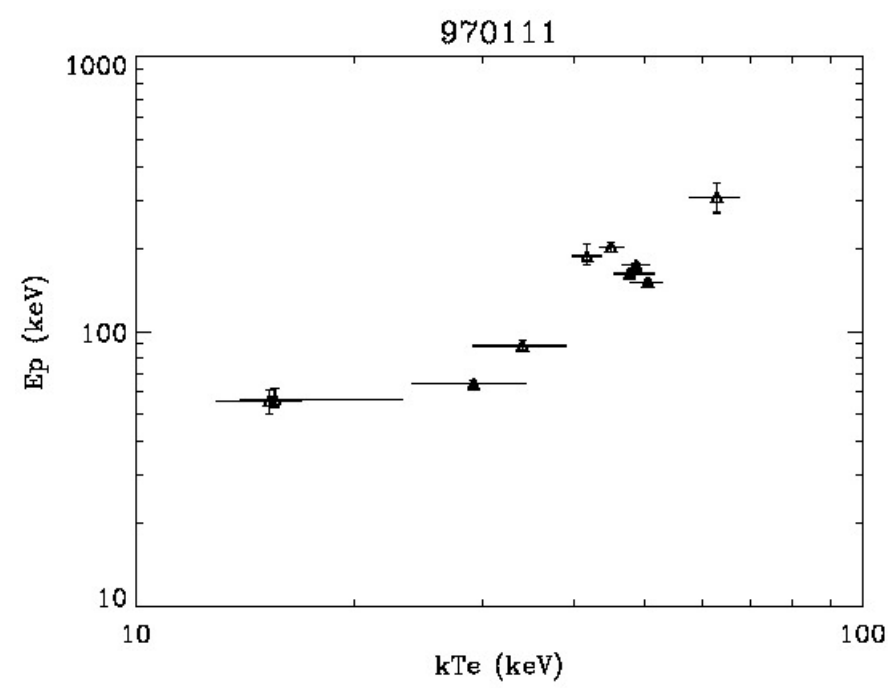

Fig. 3. Correlation between peak energy of the $E F(E)$ spectrum and the electron temperature of the plasma outflow, in the case of GRB 970111. 
Another important result is the correlation found between the seed photon temperature $k T_{s}$ and the apparent photospheric radius $R_{p h}$ (see eq. 2). In Fig. 6 it is shown an example of this result. Given its power-law shape, this correlation implies an adiabatic expansion of the seed photon region that undergoes the Comptonization process.

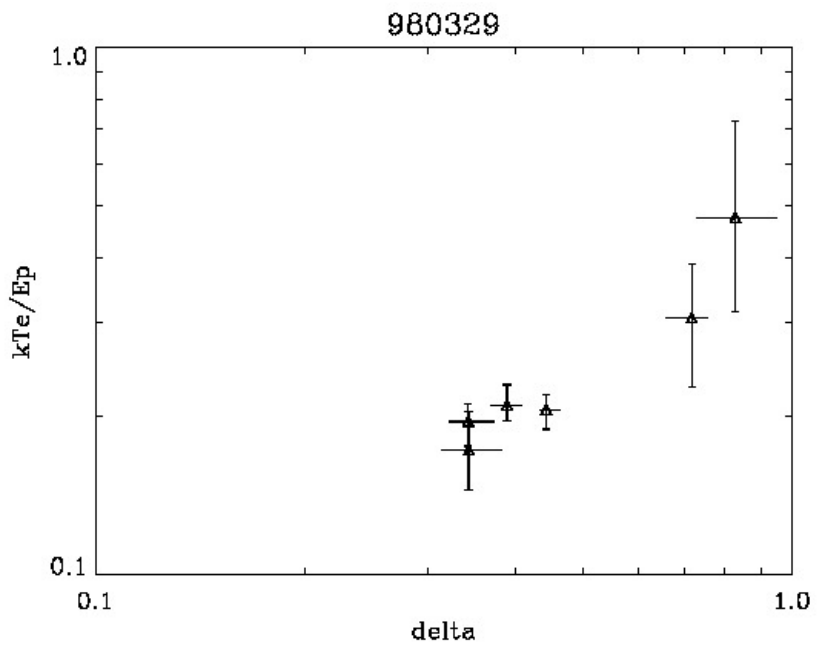

Fig. 4. Correlation between btween the $k T_{e} / E_{p}$ ratio and the bulk parameter $\delta$ of the plasma outflow, in the case of GRB 980329.

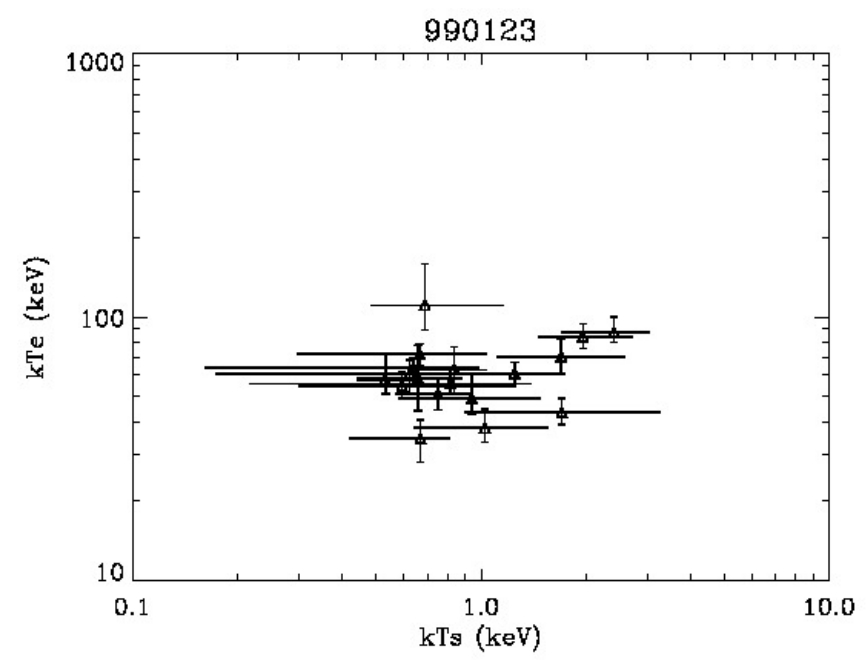

Fig. 5. Correlation between electron temperature of the plasma outflow and seed photon temperature, in the case of GRB 990123. 


\section{Conclusions}

The $E_{p}-L_{i s o}$ relation, where $L$ is the GRB luminosity ${ }^{13}$, is found valid within each GRB.

Our analysis of the 2-2000 keV time resolved spectra of the four strongest GRBs detected with BeppoSAX WFC + BATSE shows that:

- The BB + PL model proposed in Ref. ${ }^{5}$ does not provide a good description of the data. The consequences derived in Ref. 5 should be revised.

- The GRBCOMP model is found to well describe the data.

- In the GRBCOMP model, the peak energy $E_{p}$ is found to be strongly correlated with the outflow plasma temperature.

- At second order level, also the plasma outflow velocity contributes to the measured peak energy $E_{p}$ and could be responsible, along with the optical depth, of the spread in the peak energy versus flux.

- A very strong correlation has been found between seed photon temperature and photospheric radius, that is consistent with an adiabatic cooling of the region that provides the seed photons.

Definitive results will be reported in a paper now in preparation.

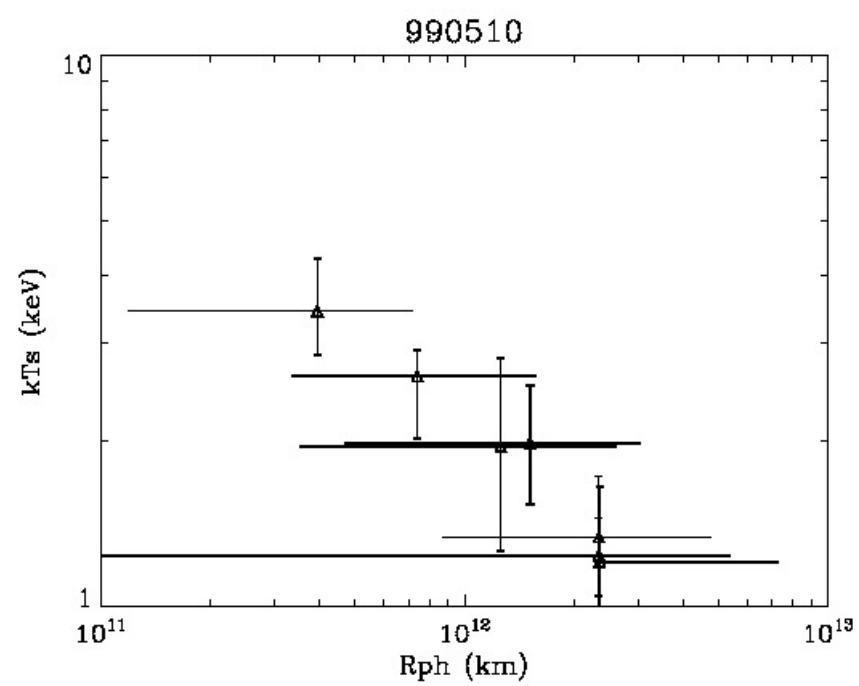

Fig. 6. Correlation between seed photon temperature and apparent photospheric radius, in the case of GRB 990510. 


\section{Acknowledgments}

We acknowledge the ASI support through the contract for high energy astrophysics studies ASI-INAF I/088/06/0, and the University of Ferrara support with FAR funds.

\section{References}

1. D. Band, J. Matteson, L. Ford, B. Schaefer, D. Palmer, B. Teegarden, T. Cline, M. Briggs, W. Paciesas, G. Pendleton, G. Fishman, C. Kouveliotou, C. Meegan, R. Wilson and P. Lestrade, Astrophys. J. 413, 281 (1993).

2. M. Tavani, Astrophys. J. 466, 768 (August 1996).

3. M. Lyutikov and R. Blandford, ArXiv Astrophysics e-prints (December 2003).

4. F. Frontera, L. Amati, E. Costa, J. M. Muller, E. Pian, L. Piro, P. Soffitta, M. Tavani, A. Castro-Tirado, D. Dal Fiume, M. Feroci, J. Heise, N. Masetti, L. Nicastro, M. Orlandini, E. Palazzi and R. Sari, Astrophys. J. Suppl. 127, 59 (March 2000).

5. F. Ryde and A. Pe'er, Astrophys. J. 702, 1211 (September 2009).

6. G. J. Fishman, C. A. Meegan, R. B. Wilson, M. N. Brock, J. M. Horack, C. Kouveliotou, S. Howard, W. S. Paciesas, M. S. Briggs, G. N. Pendleton, T. M. Koshut, R. S. Mallozzi, M. Stollberg and J. P. Lestrade, Astrophys. J. Suppl. 92, 229 (1994).

7. R. Jager, W. A. Mels, A. C. Brinkman, M. Y. Galama, H. Goulooze, J. Heise, P. Lowes, J. M. Muller, A. Naber, A. Rook, R. Schuurhof, J. J. Schuurmans and G. Wiersma, Astron. 83 Astrophys. Suppl. Ser. 125, 557 (1997).

8. A. Gal-Yam, P. Mazzali, E. O. Ofek, P. E. Nugent, S. R. Kulkarni, M. M. Kasliwal, R. M. Quimby, A. V. Filippenko, S. B. Cenko, R. Chornock, R. Waldman, D. Kasen, M. Sullivan, E. C. Beshore, A. J. Drake, R. C. Thomas, J. S. Bloom, D. Poznanski, A. A. Miller, R. J. Foley, J. M. Silverman, I. Arcavi, R. S. Ellis and J. Deng, Nature 462, 624 (2009).

9. L. Titarchuk, A. Mastichiadis and N. D. Kylafis, Astrophys. J. 487, 834 (October 1997).

10. R. Farinelli, L. Titarchuk, A. Paizis and F. Frontera, Astrophys. J. 680, 602 (June 2008).

11. P. Chardonnet, V. Chechetkin and L. Titarchuk, Astrophys. Es Space Sci. 325, 153 (February 2010).

12. L. Amati, F. Frontera, M. Tavani, J. J. M. in’t Zand, A. Antonelli, E. Costa, M. Feroci, C. Guidorzi, J. Heise, N. Masetti, E. Montanari, L. Nicastro, E. Palazzi, E. Pian, L. Piro and P. Soffitta, Astron. \& Astrophys. 390, 81 (July 2002).

13. D. Yonetoku, T. Murakami, T. Nakamura, R. Yamazaki, A. K. Inoue and K. Ioka, Astrophys. J. 609, 935 (2004). 